\title{
Posttraumatic stress disorder in interstitial cystitis/bladder pain syndrome: Relationship to patient phenotype and clinical practice implications
}

\author{
Lindsey C. McKernan PhD ${ }^{1,2}$ (D) | Benjamin N. Johnson MS $^{3}$ | \\ William S. Reynolds MD, MPH ${ }^{4} \quad$ David A. Williams PhD ${ }^{5} \quad$ Jennifer S. Cheavens PhD ${ }^{6}$ | \\ Roger R. Dmochowski MD, MMHC ${ }^{4} \quad$ Leslie J. Crofford MD $^{7}$
}

${ }^{1}$ Department of Psychiatry and Behavioral Sciences, Vanderbilt University School of Medicine, Nashville, Tennessee

${ }^{2}$ Department of Physical Medicine and

Rehabilitation, Vanderbilt University

School of Medicine, Nashville, Tennessee

${ }^{3}$ Department of Psychology, The

Pennsylvania State University, State

College, Pennsylvania

${ }^{4}$ Department of Urologic Surgery,

Vanderbilt University School of Medicine,

Nashville, Tennessee

${ }^{5}$ Department of Anesthesiology, University of Michigan, Ann Arbor, Michigan

${ }^{6}$ Department of Psychology, The Ohio

State University, Columbus, Ohio

${ }^{7}$ Department of Medicine, Vanderbilt University School of Medicine, Nashville, Tennessee

\section{Correspondence}

Lindsey C. McKernan, PhD, Departments of Psychiatry and Behavioral Sciences, Physical Medicine and Rehabilitation, Vanderbilt University Medical Center, Osher Center for Integrative Medicine, 3401 West End Avenue, Suite 380, Nashville, TN 37203.

Email: lindsey.mckernan@vanderbilt.edu

Funding information

Agency for Healthcare Research and Quality, Grant number: 6K12HS022990-

04; National Center for Advancing Translational Sciences, Grant number: UL1TR000445
Purpose: The relationship between exposure to abuse and interstitial cystitis/bladder pain syndrome (IC/BPS) is well-documented. However, studies have yet to examine posttraumatic stress disorder (PTSD), which develops following exposure to trauma and worsens health outcomes in chronic pain. We aimed to assess the prevalence and impact of PTSD in patients with IC/BPS, including their relation to genitourinary symptom presentation and widespread pain phenotype.

Materials and Methods: We recruited 202 participants with chronic pain from an academic medical center and classified 64 individuals as IC/BPS based on validated epidemiological criteria. Participants completed self-reported questionnaires assessing trauma exposure, PTSD symptoms, emotional distress, pain, and urinary symptoms. Wilcoxon rank-sum tests assessed study aims comparing IC/BPS to other chronic pain. Results: Although elevated, IC/BPS trauma exposure rates were equivalent to that of other chronic pain conditions in the sample. Despite this equivalence, in comparison, IC/ BPS patients had significantly higher rates of PTSD symptoms, with $42 \%$ meeting provisional diagnostic criteria for PTSD. Among IC/BPS, those meeting provisional criteria for PTSD had significantly higher incidence of lifetime sexual abuse, childhood trauma, and presentations consistent with the widespread pain phenotype. In IC/BPS, there was no association between PTSD and genitourinary symptoms, but provisional PTSD was associated with more pain, emotional distress, and poorer quality of life.

Conclusions: We recommend that patients with IC/BPS and widespread pain have ongoing screening and monitoring of PTSD. We recommend using trauma-informed care practices with these patients to increase trust and safety, which could improve treatment compliance and follow-up.

\section{K E Y W O R D S}

central sensitization, chronic, interstitial cystitis, painful bladder syndrome, posttraumatic stress disorders, pain 


\section{1 | INTRODUCTION}

Interstitial cystitis/bladder pain syndrome (IC/BPS) is a debilitating chronic pain condition affecting 3-8 million people ${ }^{1}$ nationally. In addition to bladder-specific symptoms, severe psychosocial symptoms can accompany IC/BPS and are associated with increased pain, disability, and polysymptomatic, poly-syndromic complaints driven by central sensitization. ${ }^{2-4}$ Leading researchers propose that individuals with widespread, systemic pain represent a distinct ("widespread") phenotype in IC/BPS compared to those with ("localized") pain confined to the bladder. ${ }^{2,3}$ Specifically, those with widespread or systemic pain have significantly worsened psychosocial burden. ${ }^{2}$ As such, treatment guidelines encourage a phenotype-directed, multimodal treatment approach incorporating medication, minimally invasive intervention, physical therapy, psychology, dietary management, and behavioral approaches to manage IC/BPS.,

Abuse exposure is recognized for its negative contribution to IC/BPS. A recent meta-analysis ${ }^{4}$ reported syndromespecific consequences of trauma exposure in IC/BPS patients. For instance, sexual abuse exposure has been related to greater sensory pain and fewer urinary symptoms, although some report this relationship as modest. A recent study ${ }^{7}$ indicated that women exposed to trauma in childhood by close others had more anxiety, dissociative tendencies, and increased anesthetic bladder capacity, proposing that psychosocial factors may drive symptoms in this subset of patients. ${ }^{8}$

Despite well-known associations between IC/BPS and abuse exposure, there has yet to be an investigation into the prevalence and impact of posttraumatic stress disorder (PTSD) in IC/BPS. A common misconception equates exposure to trauma with PTSD. Epidemiological data indicate that while up to $90 \%$ of Americans experience some form of trauma of variable severity in their lives, only $8.3 \%$ will go on to develop PTSD. ${ }^{9}$ However, the effects of PTSD can be catastrophic. According to the American Psychiatric Association, PTSD is a psychiatric disorder that develops following exposure to a traumatic event, marked by persistent hyperarousal and reactivity, re-experiencing, avoidance of trauma-related stimuli, and negative alteration in cognition and mood for 1 month or more. Symptoms can develop immediately, have delayed onset, and recur at different points when activated by an event that serves as a reminder of the initial trauma. In chronic pain conditions that share similar characteristics to IC/BPS, PTSD is associated with heightened evoked pain responding and hyper-reactivity to noxious stimuli leading to increased chronicity and worsened clinical presentation. ${ }^{10,11}$ PTSD and chronic pain are theorized to maintain each other through shared mechanisms, such as chronic hyperarousal. ${ }^{10}$

To date, we have yet to understand the role that PTSD plays in IC/BPS, even though it may have significant clinical and research implications, as urologic care may activate PTSD symptoms. Further, we do not know if PTSD worsens symptom presentation in IC/BPS or how PTSD relates to IC/ BPS phenotypes (eg, "localized" vs "widespread"2,3).

To explore how PTSD may affect individuals with IC/ BPS, we sought to examine the association between PTSD and IC/BPS, including how this may differ from individuals with non-IC/BPS chronic pain in a convenience sample of individuals with chronic pain. Second, we sought to understand how provisional PTSD diagnosis relates to clinical presentation. We hypothesized that (1) individuals with IC/BPS would have higher rates of both trauma exposure and PTSD, and that (2) those with both IC/BPS and PTSD would exhibit worse health outcomes when compared to participants with IC/BPS only. Finally, we (3) examined characteristics of PTSD and pain associated with polysymptomatic, poly-syndromic complaints (ie, "widespread phenotype") in IC/BPS.

\section{2 | MATERIALS AND METHODS}

The institutional review board reviewed and approved study procedures. We recruited consenting participants with chronic pain $(N=211)$ to complete a series of validated questionnaires (online at home, by paper, or at an on-site computer station) assessing physical symptoms, emotional symptoms, and trauma-related experiences from 1/2016-3/ 2017. We identified participants through either a large university-affiliated medical outpatient clinic, a hospitalwide research listserv, or via online advertisement through ResearchMatch (Figure 1). ${ }^{12}$ Eligible participants were English-speaking adults $(>18)$, and had a "yes" response from either the patient or referring medical provider to the question "do you have a medical diagnosis involving chronic pain?" (for 6 months or longer). We confirmed the presence of chronic pain via electronic health record review. Exclusionary criteria included diagnosis of cognitive or thought disorder, current substance dependence, or active suicidal ideation. In total, 202 participants $\left(M_{\mathrm{age}}=44.89,79.7 \%\right.$ female) completed the study and comprised the analyzed sample. As we recruited our sample through the larger hospital community primarily outside of urology specialization, we defined a subset of participants with IC/BPS $(n=64)$ using RAND Interstitial Cystitis Epidemiologic ( $\mathrm{RICE}^{1}$ ) sensitivity criteria, which requires patients to report 1) pain, pressure, and discomfort in the pelvic area; 2) daytime frequency of $>10$; and 3) urinary urgency due to pain, pressure, or discomfort (and not fear of wetting). Previous investigations ${ }^{13}$ found that when compared to a clinical cohort, individuals meeting RICE sensitivity criteria exhibited remarkably similar demographic and symptom profiles, indicating the utility of this approach. 


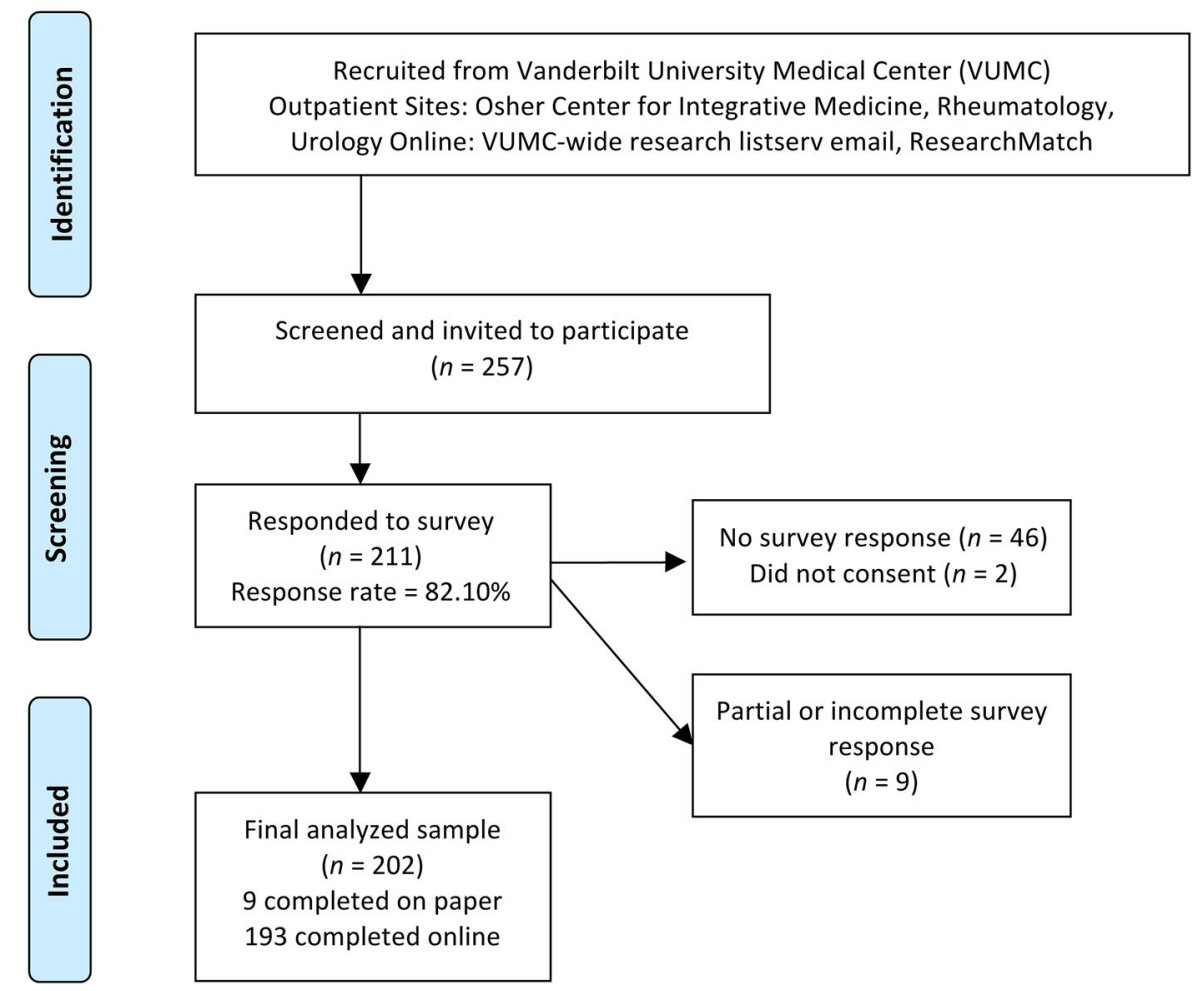

FIGURE 1 Study recruitment

\section{1 | Measures}

To identify IC/BPS, we used the RICE high sensitivity ${ }^{1}$ definition (91\% sensitivity, 42\% specificity). We assessed PTSD with the PTSD-Checklist-5 ( PCL $\left.^{14}\right)$, a 20-item self-report measure corresponding to DSM-5 PTSD diagnostic criteria established by the American Psychiatric Association. We assigned provisional PTSD diagnosis using DSM-5 PTSD diagnostic criterion rules. ${ }^{15} \mathrm{We}$ assessed childhood trauma exposure with the Childhood Abuse and Trauma Scale $\left(\right.$ CATS $\left.^{16}\right)$ and lifetime trauma exposure with the Trauma History Questionnaire $\left(\mathrm{THQ}^{17}\right)$. Given the differing effects of child trauma ${ }^{7}$ in IC/ BPS patients, we included an adjusted THQ score for adult trauma only by removing all traumas prior to age 18 .

Regarding pain, we used the Numeric Rating Scale (NRS$11^{18}$ ) to assess current pain, Michigan Body Map (MBM $\left.{ }^{19}\right)$ for widespread pain, and central sensitization inventory $\left(\mathrm{CSI}^{20}\right)$. CSI items 11 (dysuria), 21 (urinary frequency), and 25 (pelvic pain) examined IC/BPS-specific symptoms. We characterized participants as having likely central sensitization (CS) by a validated total cutoff score of $\geq 40$, which discriminates CS from non-CS pain populations. ${ }^{21}$ We used this cutoff to differentiate individuals with likely widespread, systemic pain for our third aim, in which we examined characteristics of PTSD and pain associated with the widespread phenotype in IC/BPS. Last, the Hospital Anxiety and Depression Scale $\left(\mathrm{HADS}^{22}\right)$ captured affective distress and Satisfaction With Life Scale $\left(\right.$ SWLS $\left.^{23}\right)$ quality of life. All measures constitute valid and reliable assessments used across previous investigations in IC/BPS and non-cancer chronic pain to assess pain and psychosocial functioning.

\subsection{Data analysis}

We conducted analyses using SPSS 25. Due to positive skew, we used Wilcoxon rank-sum tests to compare outcome variables by groups for each hypothesis. This included examining PTSD and trauma characteristics in IC/BPS versus other chronic pain groups (Hypothesis 1); within the IC/BPS group, comparing those with and without PTSD (Hypothesis 2 ); and when exploring differences between IC/BPS + CS versus IC/BPS-only groups (Hypothesis 3 ). We assigned group membership based on validated clinical cutoffs along variables of interest (RICE, PCL, CSI). We used chi-square analysis with Fisher's exact test to compare frequencies among categorical variables (eg, gender) by groups. To adjust for multiple comparisons, we used alpha of $P<0.01$ (Bonferroni adjustment: $0.05 / 5=0.01$ ) to define statistical significance.

\section{3 | RESULTS}

\section{1 | Sample characteristics}

The sample (Table 1) was largely female (79.7\%) and white (81.2\%), with diverse educational and vocational status. Men were significantly older than women $\left(M_{\mathrm{men}}=49.8\right.$, $M_{\text {women }}=43.8$ ). Women endorsed higher levels of 
TABLE 1 Demographic characteristics by presence or absence of IC/BPS

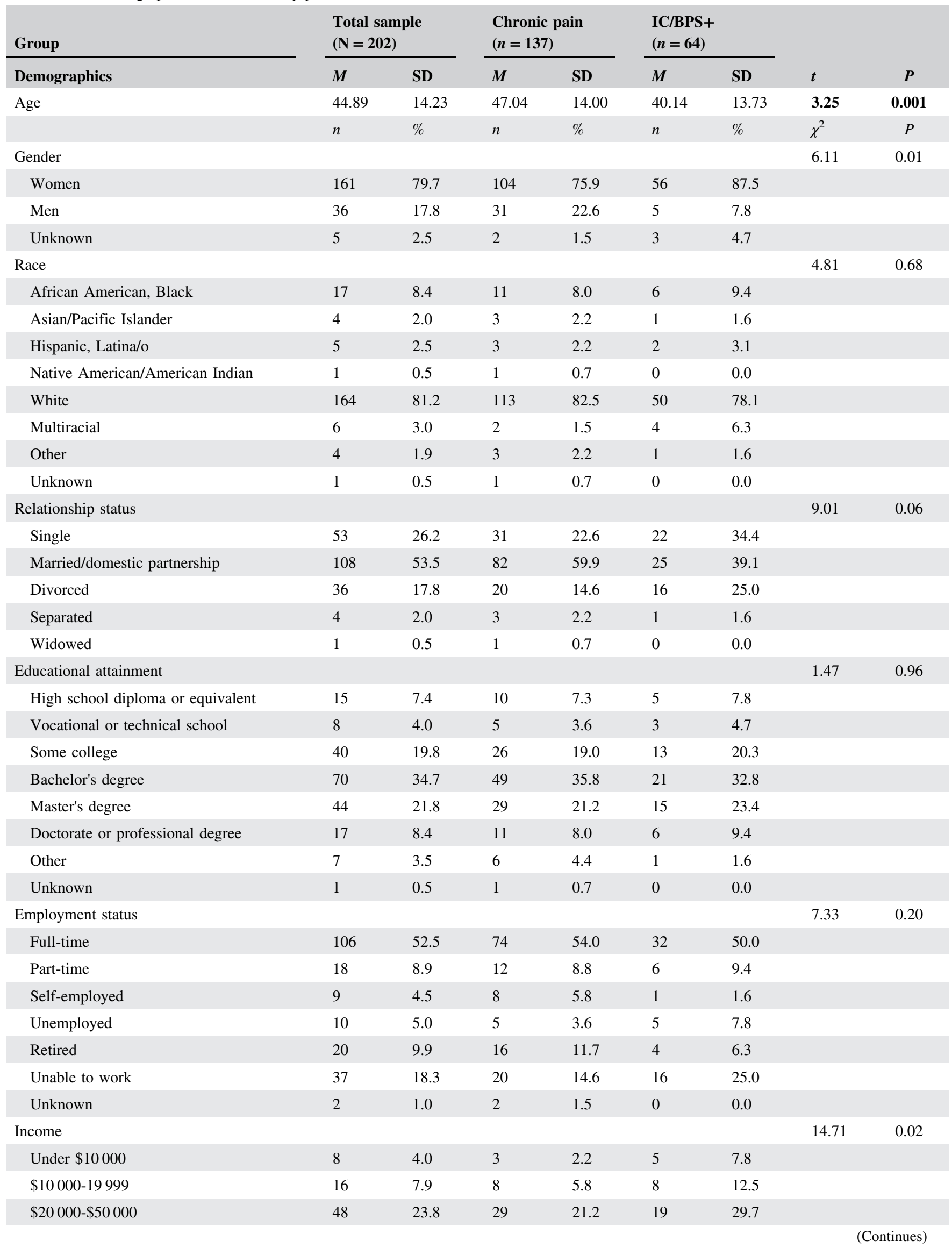


TABLE 1 (Continued)

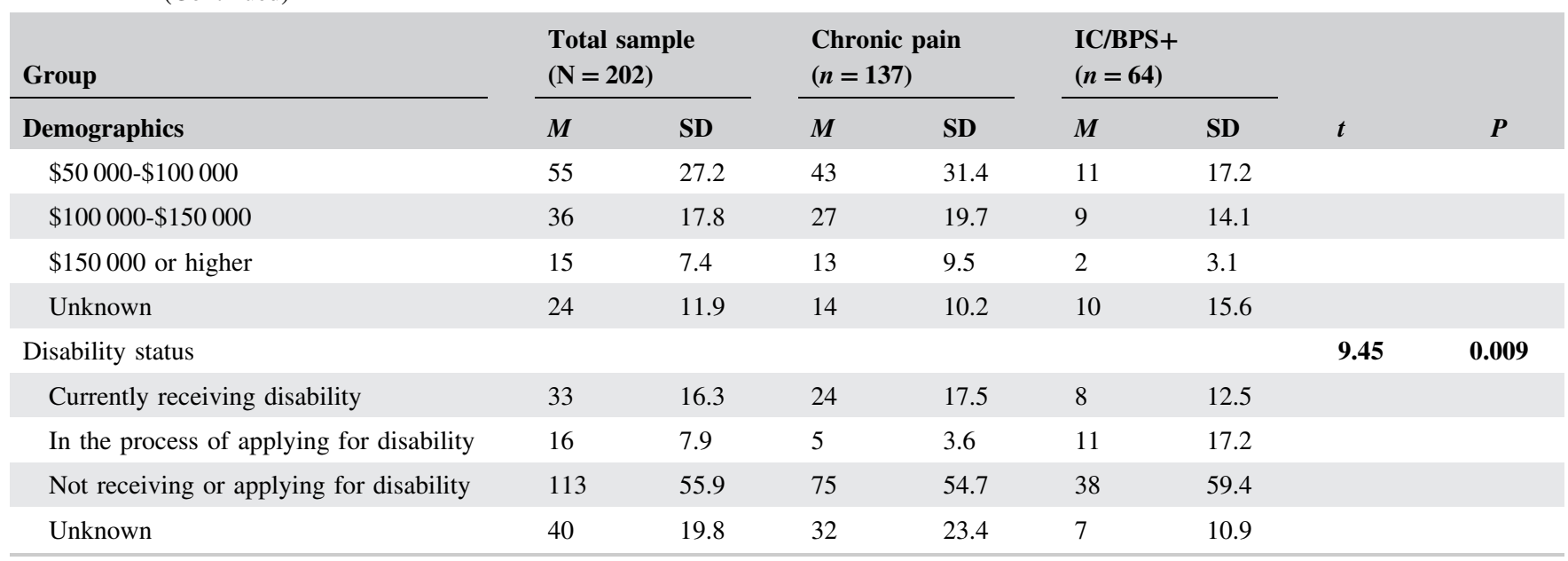

$M$, mean; SD, standard deviation; $n$, frequency of demographic characteristic; $\%$, percentage of subgroup. Statistical tests evaluate differences in distributions of each demographic variable by IC/BPS group. Fisher's exact test $P$-values reported (two-tailed test) for all chi-square analyses. Significant tests at $P<0.01$ are denoted by bold font.

widespread pain (MBM: $z=3.12, P=0.002)$, total central sensitization (CSI: $z=2.91, P=0.004)$, and dysuria $(z=3.05$, $P=0.002)$ than men. Women reported higher rates of childhood neglect (CATS: $z=-2.01, P=0.045$ ), while men endorsed more general disaster/traumas (THQ: $z=-2.01$, $P=0.045)$. Men and women did not differ on other variables.

\subsection{Trauma exposure and PTSD in IC/BPS}

Patients with likely IC/BPS were significantly younger than those with other forms of chronic pain and displayed higher levels of central sensitization, PTSD symptoms, anxiety, and depression (Table 2). Patients with likely IC/BPS did not differ in exposure to trauma in childhood or adulthood when compared to other chronic pain.

\section{3 | PTSD and health outcomes in IC/BPS}

Patients with co-occurring IC/BPS and provisional PTSD reported poorer health functioning broadly. The co-occurrence group endorsed higher levels of current pain, central sensitization, childhood trauma, lifetime physical/sexual trauma, anxiety, depression, and quality of life than IC/ BPS patients without PTSD (Table 3). IC/BPS patients with PTSD did not differ in reported dysuria, pelvic pain, frequency, or on the remaining variables.

\subsection{Widespread pain phenotype in IC/BPS}

Patients with co-occurring IC/BPS and CS endorsed higher levels of current and widespread pain, PTSD symptoms, and all forms of emotional distress than IC/BPS patients without CS (Table 4). The groups did not differ on other variables. Given the similarity between IC/BPS + PTSD and IC/BPS + CS groups, we examined overlap between group memberships
(Figure 2); all individuals in the IC/BPS + PTSD group fell into the IC/BPS + CS group (25 of 51 individuals), while only $50 \%$ of IC/BPS + CS patients met criteria for provisional PTSD (similar to the $42 \%$ rate for IC/BPS alone). Notably, this overlap was consistent across all domains of PTSD symptoms.

\section{4 | DISCUSSION}

In this cohort of individuals with chronic pain conditions, individuals with IC/BPS did not differ in their lifetime exposure to trauma, including sexual abuse and physical abuse, compared to those with other chronic pain. However, in spite of equivalent trauma exposure, participants with IC/BPS exhibited significantly higher rates of PTSD symptoms and psychosocial distress. This suggests that it may not be trauma exposure, but individuals' reactions to trauma that are a source of distress for this population.

When examining the IC/BPS cohort separately, $42 \%$ of individuals with IC/BPS met provisional PTSD criteria. Not only is this roughly five times higher than reported in the general population, ${ }^{9}$ it is two times higher than non-IC chronic pain participants in our sample (23\%). A recent systematic review ${ }^{24}$ also found that certain pain subtypes (eg, fibromyalgia, low back pain) have significantly elevated PTSD rates. Furthermore, individuals with IC/BPS and cooccurring provisional PTSD reported higher rates of pain, psychosocial distress, greater exposure to lifetime sexual or physical abuse, and childhood trauma. Although overall pain levels were higher, PTSD was not associated with an altered IC/BPS presentation with respect to urinary urgency, frequency, or pelvic pain. This negative finding coincides with previous reports of the "widespread" IC/BPS phenotype, where patients have higher rates of polysymptomatic complaints, psychosocial distress, and pain levels but do not differ based on urinary symptoms. ${ }^{2}$ 
TABLE 2 Pain, trauma, and distress by IC/BPS and other chronic pain groups

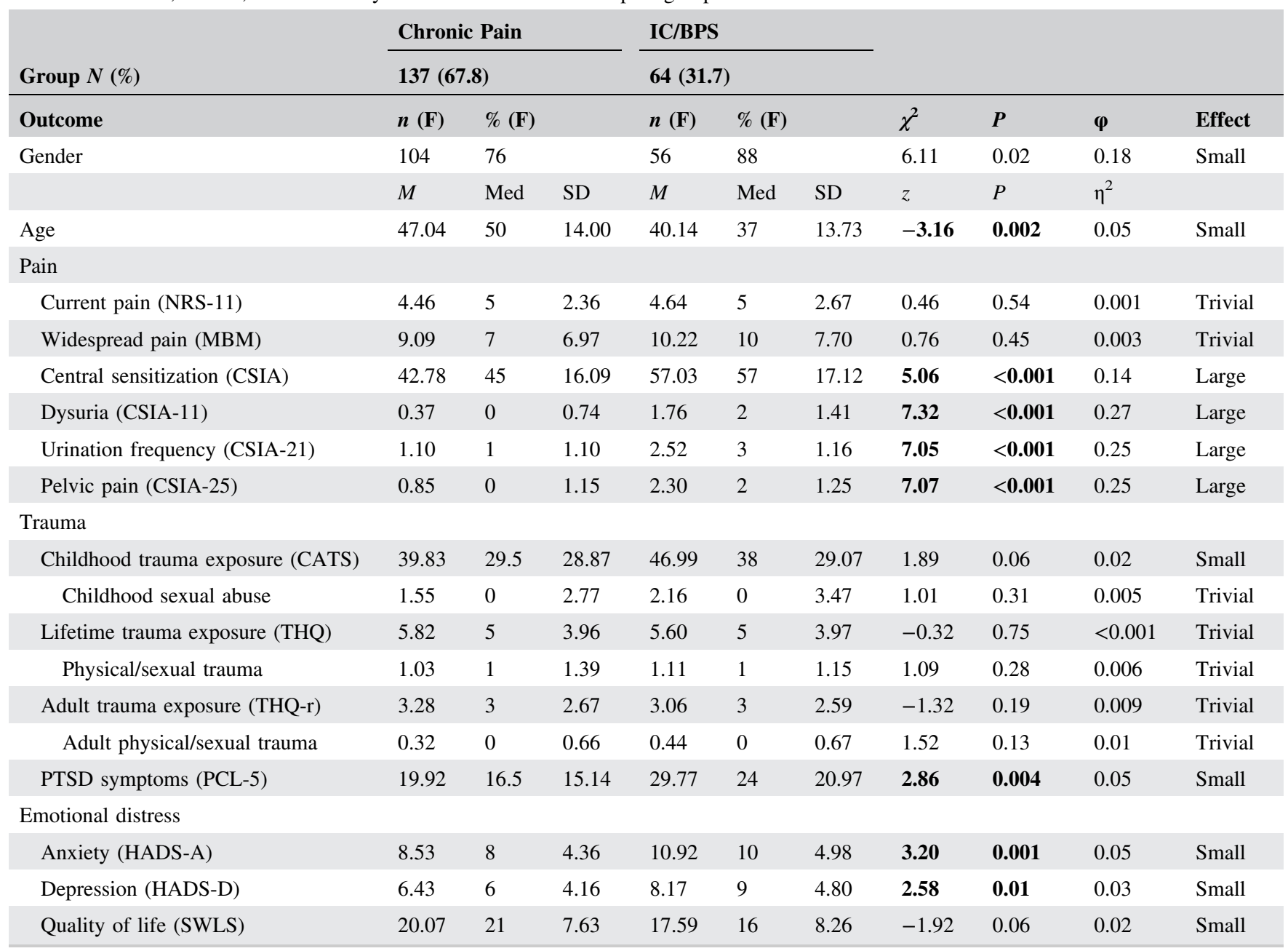

All group comparisons conducted using the nonparametric Wilcoxon rank-sum test, except gender. Group comparisons on gender conducted using Chi-square contingency table analysis with Fisher's exact test. Significant tests at $P<0.01$ are denoted by bold font. IC/BPS, interstitial cystitis/bladder pain syndrome; F, female; $M$, mean; Med, median; SD, standard deviation; NRS, numeric rating scale; MBM, Michigan body map; CSIA, central sensitization inventory—Part A; CATS, child abuse and trauma scale; THQ, trauma history questionnaire; THQ-r, revised THQ after removing childhood trauma exposure; PTSD, posttraumatic stress disorder; PCL, PTSD Checklist for DSM-5; PCS, pain catastrophizing scale; HADS, hospital anxiety and depression scale; SWLS, satisfaction with life scale.

In this sample, IC/BPS patients exposed to childhood abuse were more likely to meet criteria for PTSD. While childhood sexual abuse contributes, it does not fully explain the effect, and other aspects of negative or hostile home environments play a role. Since relational or childhood trauma by close others significantly impacts patient psychosocial and bladder functioning, this prompts further investigation. Further, although they did not formally assess PTSD, Chiu et $\mathrm{al}^{7}$ found that dissociation, characteristic of PTSD, was associated with childhood trauma. Together, this may provide preliminary evidence of a relevant aspect in clinical profiles of a subset of IC/BPS patients.

We assessed whether individuals with IC/BPS and provisional PTSD exhibited characteristics of the "widespread" IC/BPS phenotype-polysymptomatic, polysyndromic complaints suggesting central sensitization (CS). Though not significantly linked to specific trauma exposure, the widespread phenotype was associated with higher levels of both current and widespread pain (a median of 12 pain sites compared to 1 pain site) and greater PTSD symptoms and psychological impairment. All individuals with IC/BPS + PTSD fell within the widespread phenotype, which comprised roughly $50 \%$ of the group, suggesting that over and above trauma exposure, PTSD is uniquely related to this phenotypic presentation of IC/BPS. However, given the small size of the comparison group (with no CS), further research is needed to confirm this finding.

\subsection{Clinical practice implications}

International guidelines ${ }^{5}$ recommend regular and ongoing assessment of the negative psychological consequences of IC/BPS and psychological distress in relation to pain. As little information exists about the management of PTSD in relation to patient presentation, evaluation, and management in IC/BPS (ie, phenotype-directed treatment ${ }^{5}$ ), we seek to 
TABLE 3 Pain, trauma, and distress by IC/BPS and IC/BPS plus PTSD groups

\begin{tabular}{|c|c|c|c|c|c|c|c|c|c|c|}
\hline \multirow{2}{*}{$\frac{\text { Group } N(\%)}{\text { Outcome }}$} & \multicolumn{3}{|c|}{$\begin{array}{l}\text { IC/BPS only } \\
35(17.3) \\
\end{array}$} & \multicolumn{3}{|c|}{$\begin{array}{l}\text { IC/BPS + PTSD } \\
25(12.4)\end{array}$} & \multirow[b]{2}{*}{$\chi^{2}$} & \multirow[b]{2}{*}{$P$} & \multirow[b]{2}{*}{$\varphi$} & \multirow[b]{2}{*}{ Effect } \\
\hline & $n(\mathbf{F})$ & \multicolumn{2}{|l|}{$\%(\mathbf{F})$} & $n(\mathbf{F})$ & \multicolumn{2}{|c|}{$\%(\mathbf{F})$} & & & & \\
\hline \multirow{2}{*}{ Gender } & 31 & 86 & & 21 & 85 & & 0.00 & 1.00 & 0.00 & Trivial \\
\hline & $M$ & Med & $\mathrm{SD}$ & $M$ & Med & SD & $z$ & $P$ & $\eta^{2}$ & \\
\hline Current pain (NRS-11) & 3.89 & 4 & 2.83 & 5.84 & 6 & 2.08 & 2.68 & 0.007 & 0.12 & Medium \\
\hline Widespread pain (MBM) & 9.26 & 9 & 7.9 & 11.48 & 13 & 7.48 & 1.23 & 0.22 & 0.03 & Small \\
\hline Central sensitization (CSIA) & 49.33 & 53 & 16.06 & 67.5 & 67.5 & 13.58 & 3.84 & $<0.001$ & 00.26 & Large \\
\hline Dysuria (CSIA-11) & 1.65 & 2 & 1.32 & 1.76 & 2 & 1.54 & 0.19 & 0.85 & $<0.001$ & Trivial \\
\hline Childhood trauma exposure (CATS) & 33.62 & 25.33 & 23.27 & 64.63 & 66 & 26.14 & 4.13 & $<0.001$ & 0.29 & Large \\
\hline Childhood sexual abuse & 1.29 & 0 & 2.72 & 3.36 & 2 & 4.07 & 2.55 & 0.01 & 0.11 & Medium \\
\hline Lifetime trauma exposure (THQ) & 4.40 & 4 & 2.89 & 7.08 & 7 & 4.10 & 2.49 & 0.01 & 0.10 & Medium \\
\hline Physical/sexual trauma & 0.69 & 0 & 0.87 & 1.71 & 2 & 1.12 & 3.50 & $<0.001$ & 0.21 & Large \\
\hline Adult trauma exposure (THQ-r) & 2.60 & 3 & 1.99 & 3.68 & 3 & 2.87 & 1.36 & 0.18 & 0.03 & Small \\
\hline Physical/sexual trauma & 0.34 & 0 & 0.59 & 0.54 & 0 & 0.72 & 1.11 & 0.27 & 0.02 & Small \\
\hline PTSD symptoms (PCL-5) & 14.97 & 15 & 11.27 & 48.72 & 45 & 14.01 & 6.07 & $<0.001$ & 0.66 & Large \\
\hline \multicolumn{11}{|l|}{ Emotional distress } \\
\hline
\end{tabular}

All group comparisons conducted using the nonparametric Wilcoxon rank-sum test, except gender. Group comparisons on gender conducted using Chi-square contingency table analysis with Fisher's exact test. Significant tests at $P<.01$ are denoted by bold font. IC/BPS, interstitial cystitis/bladder pain syndrome; PTSD, posttraumatic stress disorder; F, female; $M$, mean; Med, median; SD, standard deviation; NRS, numeric rating scale; MBM, Michigan body map; CSIA, central sensitization inventory—Part A; CATS, child abuse and trauma scale; THQ, trauma history questionnaire; THQ-r, revised THQ after removing childhood trauma exposure; PCL, PTSD checklist for DSM5; PCS, pain catastrophizing scale; HADS, hospital anxiety and depression scale; SWLS, satisfaction with life scale.

offer suggestions for practice that may be beneficial to patient-provider interactions, treatment, and the patient experience. We suggest the following principles of trauma informed $\mathrm{care}^{25}$ in the treatment of IC/BPS. They emphasize strong patient-centered communication and behavioral techniques to establish patient-provider trust and rapport, reduce anxiety, and increase perceived control during appointments:

PTSD Screening: Previous studies indicate that individuals with IC/BPS report abuse at significantly lower rates when asked directly by medical providers. ${ }^{26}$ Consequently, we recommend initially assessing PTSD through monitoring specific symptoms over direct questioning (eg, hyper-arousal, nightmares), specifically for patients with widespread pain presentations given its overlap with PTSD.

Initial appointment and ongoing management: Encouraging trauma survivors to collaborate in their appointments may lead to higher perceived control and trust. ${ }^{25}$ We suggest offering patients an overview of what will happen during the initial visit, including procedures involved. We provide suggestions to enhance perceived safety during examination or procedures in Table 5. Pelvic floor physical therapy (PFPT) can trigger PTSD symptoms. ${ }^{27}$ If a patient has PTSD and is emotionally distressed, PFPT may be contra-indicated until the patient has adequate support.

\subsection{Study strengths and limitations}

This is the first study to our knowledge using validated measures to assess PTSD symptoms and likely PTSD diagnoses in IC/BPS, while also considering trauma exposure. Along with prevalence, this assessment enabled us to differentiate whether PTSD symptoms, not just trauma exposure, were associated with IC/BPS group membership. We used a 
TABLE 4 Pain, trauma, and distress by IC/BPS and IC/BPS plus central sensitization (CS) groups

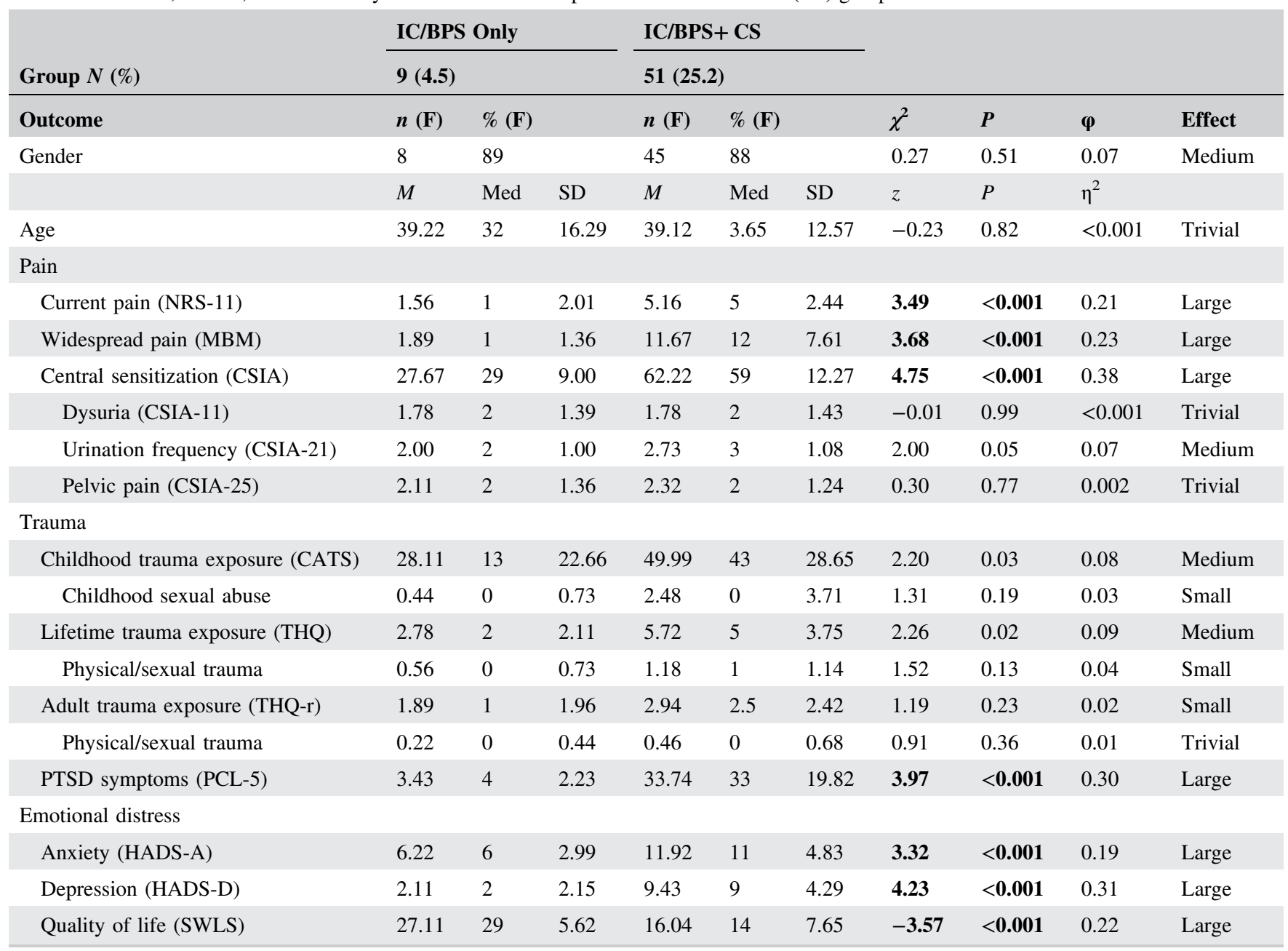

All group comparisons conducted using the nonparametric Wilcoxon rank-sum test, except gender. Group comparisons on gender conducted using Chi-square contingency table analysis with Fisher's exact test. Significant tests at $P<0.01$ are denoted by bold font. IC/BPS, interstitial cystitis/bladder pain syndrome; CS, central sensitization; F, female; $M$, mean; Med, median; SD, standard deviation; NRS, numeric rating scale; MBM, Michigan body map; CSIA, central sensitization inventory—Part A; CATS, child abuse and trauma scale; THQ, trauma history questionnaire; THQ-r, revised THQ after removing childhood trauma exposure; PTSD, posttraumatic stress disorder; PCL, PTSD checklist for DSM-5; PCS, pain catastrophizing scale; HADS, hospital anxiety and depression Scale; SWLS, satisfaction with life scale.

clinical sample with a chronic pain comparison group, which may increase validity of our findings. We used validated clinical cutoffs consistent with PTSD and medical diagnoses to improve accuracy, clinical applicability, and reproducibility of this work.

Study limitations include reliance on a single major academic medical center and cross-sectional data. We did not collect information on symptom duration beyond 6 months. Our IC/BPS group sample size was relatively small, especially those without concurrent CS $(n=9)$, potentially leading to decreased power in group comparisons. However, this is reasonable given the low prevalence of IC/BPS. Also our IC/BPS group was younger and more likely female than the other chronic pain group; although this demographic composition mirrors population data on IC/BPS and recent investigations, ${ }^{7}$ group demographic differences may confound results. We used epidemiological criteria to identify likely cases of IC/BPS, as the majority of patients were seen outside of urology. Future investigations would reduce the potential for misclassification with formal evaluation by a urologist. We assessed bladder-specific symptoms between groups with specific CSI items. We would have preferred to use in-depth scales validated for this reason specifically. Information bias limits retrospective recall of traumatic events. While a diagnostic clinical interview is preferred in PTSD assessment, investigations show marked variability in participants' willingness to acknowledge abuse exposure in person and this may remain a standing limitation in this line of research without longitudinal study. Furthermore, although growing evidence indicates the reliability and validity of online questionnaire administration, reliance on online self-report surveys may limit the clinical applicability of our results. Lastly, trauma-informed care practice guidelines have yet to be studied in IC/BPS. 


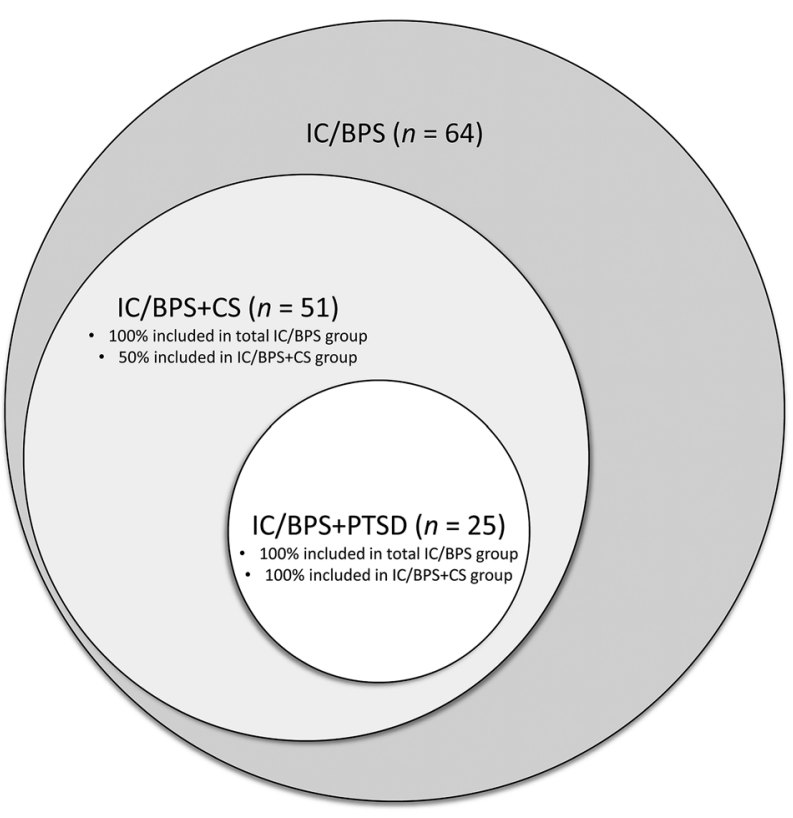

FIGURE 2 Proportion of IC/BPS sample with central sensitization (CS) and posttraumatic stress disorder (PTSD)

\section{3 | Future research}

Replication and extension of these findings, particularly in terms of PTSD prevalence, is needed with larger clinical samples. Future investigations may explore the role of PTSD from childhood trauma in IC/BPS symptom development and maintenance, using structured diagnostic assessment tools. As PTSD symptom reduction in similar chronic pain conditions can lead to pain reduction and improved outcomes, it may be a fruitful area of investigation in clinical trials.

\section{5 | CONCLUSIONS}

Although individuals with IC/BPS did not differ in their exposure to abuse compared to other chronic pain conditions, those with IC/BPS had significantly higher likelihood of PTSD, particularly when exposed to childhood trauma. Our investigation highlights the high potential for PTSD in IC/BPS (42\%) and the strong associations between concurrent IC/BPS and PTSD and both symptoms and quality of life. PTSD also appears associated with the "widespread" pain phenotype and merits consideration for multimodal treatment in these patients.

\section{ACKNOWLEDGMENTS}

We would like to acknowledge the American Psychological Association Society of Clinical Psychology (Division 12) Research Assistance Task Force for facilitating this work. This manuscript was prepared with support from the Vanderbilt Patient Centered Outcomes Research Education and Training Initiative and the Agency for Healthcare Research and Quality (AHRQ 6K12HS022990-04) and by Vanderbilt University Medical Center CTSA award No. UL1TR000445 from the National Center for Advancing Translational Sciences. Its contents are solely the responsibility of the authors and do not necessarily represent official views of the National Center for Advancing Translational Sciences or the National Institutes of Health.

TABLE 5 Trauma-informed care practices as applied to patient examination procedures (adapted from Elliot et $\mathrm{al}^{25}$ )
Prior to examination ${ }^{\mathrm{a}}$
- Obtain consent: provide patient with a choice of whether or not to proceed with exam, and medical rationale for why it may be helpful
- Provide details and parameters of exam, such as specific procedures, timeframe, duration, and objective
- Ask if patient would like a support person present
- Ask if steps can be taken to increase comfort
- Provide patient with choice about clothing, body position, use of stirrups
- Establish a verbal (word) or non-verbal (foot wiggle, arm raising) signal to indicate stopping the exam, when medically possible
- Allow patient to ask questions and clarify as able
- Develop a plan of active coping techniques to use during the examination (eg, deep breathing)

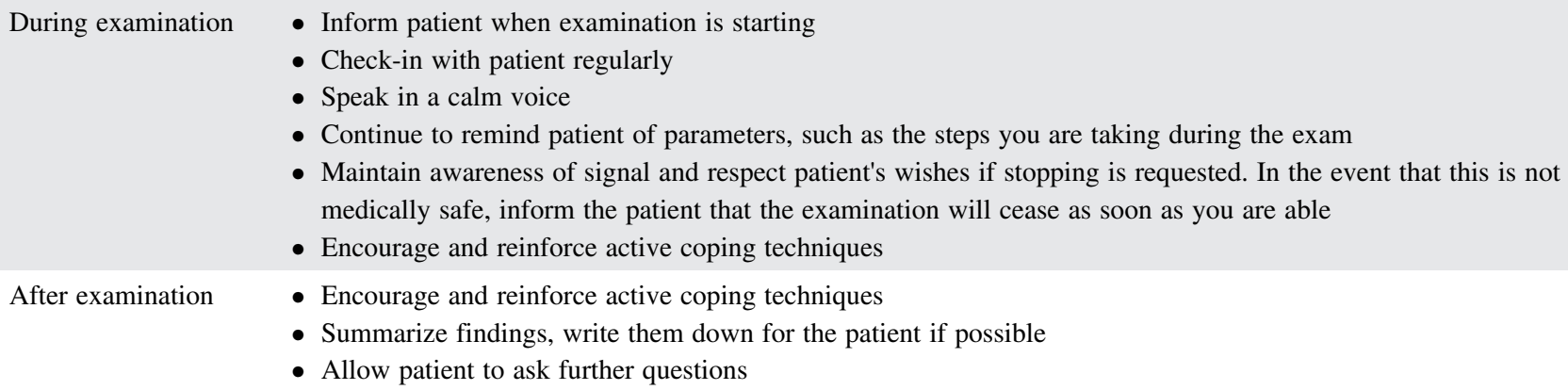

${ }^{a}$ The care practices suggested above in examinations are interchangeable with diagnostic testing, treatment, and procedures. 


\section{ORCID}

Lindsey C. McKernan (iD http://orcid.org/0000-0001-94198502

\section{REFERENCES}

1. Berry SH, Elliott MN, Suttorp M, et al. Prevalence of symptoms of bladder pain syndrome/interstitial cystitis among adult females in the United States. J Urol. 2011;186:540-544.

2. Lai HH, Jemielita T, Sutcliffe S, et al. Characterization of whole body pain in urological chronic pelvic pain syndrome at baseline: a MAPP research network study. J Urol. 2017;198:622-631.

3. Nickel JC, Shoskes D, Irvine-Bird K. Clinical phenotyping of women with interstitial cystitis/painful bladder syndrome: a key to classification and potentially improved management. J Urol. 2009;182:155-160.

4. McKernan LC, Walsh CG, Reynolds WS, Crofford LJ, Dmochowski RR, Williams DA. Psychosocial co-morbidities in interstitial cystitis/bladder pain syndrome (IC/BPS): a systematic review. Neurourol Urodyn. 2018;37:926-941.

5. European Association of Urology. (2017). Guidelines: 2017 Edition. Available online at: http://uroweb.org/wp-content/ uploads/Guidelines_WebVersion_Complete-1.pdf.

6. Dellis AE, Papatsoris AG. Bridging pharmacotherapy and minimally invasive surgery in interstitial cystitis/bladder pain syndrome treatment. Expert Opin Pharmacother. 2018;19:1369-1373. https://doi.org/10.1080/14656566.2018. 1505865.

7. Chiu C-D, Lee M-H, Chen W-C, Ho HL, Wu H-C. Childhood trauma perpetrated by close others, psychiatric dysfunction, and urological symptoms in patients with interstitial cystitis/bladder pain syndrome. J Psychosom Res. 2017;93:90-95.

8. Chiu CD, Lee MH, Chen WC, Ho HL, Wu HC. Alexithymia and anesthetic bladder capacity in interstitial cystitis/bladder pain syndrome. J Psychosom Res. 2017;100:15-21.

9. Kilpatrick DG, Resnick HS, Milanak ME, Miller MW, Keyes KM, Friedman MJ. National estimates of exposure to traumatic events and PTSD prevalence using DSM-IV and DSM-5 criteria. J Trauma Stress. 2013;26:537-547.

10. Kimerling R, Clum GA, Wolfe J. Relationships among trauma exposure, chronic posttraumatic stress disorder symptoms, and selfreported health in women: replication and extension. $J$ Trauma Stress. 2000;13:115-128.

11. Sherman AL, Morris MC, Bruehl S, Westbrook TD, Walker LS. Heightened temporal summation of pain in patients with functional gastrointestinal disorders and history of trauma. Ann Behav Med. 2015;49:785-792.

12. Harris PA, Scott KW, Lebo L, Hassan N, Lighter C, Pulley J. Research Match: a national registry to recruit volunteers for clinical research. Acad Med. 2012;87:66.

13. Konkle KS, Berry SH, Elliott MN, et al. Comparison of an interstitial cystitis/bladder pain syndrome clinical cohort with symptomatic community women from the RAND Interstitial Cystitis Epidemiology study. J Urol. 2012;187:508-512.

14. Blevins CA, Weathers FW, Davis MT, Witte TK, Domino JL. The posttraumatic stress disorder checklist for DSM-5 (PCL-5): Development and initial psychometric evaluation. J Trauma Stress. 2015;28:489-498.
15. Wortmann JH, Jordan AH, Weathers FW, et al. Psychometric analysis of the PTSD Checklist-5 (PCL-5) among treatment-seeking military service members. Psychol Assess. 2016;28: 1392-1403.

16. Sanders B, Becker-Lausen E. The measurement of psychological maltreatment: early data on the child abuse and trauma scale. Child Abuse Negl. 1995;19:315-323.

17. Hooper LM, Stockton P, Krupnick JL, Green BG. Development, use, and psychometric properties of the Trauma History Questionnaire. J Loss Trauma. 2011;16:258-283.

18. Farrar JT, Young JP, LaMoreaux L, Werth JL, Poole RM. Clinical importance of changes in chronic pain intensity measured on an 11point numerical pain rating scale. Pain. 2001;94:149-158.

19. Brummett CM, Bakshi RR, Goesling J, et al. Preliminary validation of the michigan body map (MBM). Pain. 2016;157:1205-1212.

20. Mayer TG, Neblett R, Cohen H, et al. The development and psychometric validation of the central sensitization inventory. Pain Pract. 2012;12:276-285.

21. Neblett R, Hartzell MM, Cohen H, et al. Ability of the central sensitization inventory to identify central sensitivity syndromes in an outpatient chronic pain sample. Clin J Pain. 2015;31:323-332.

22. Zigmond AS, Snaith RP. The hospital anxiety and depression scale. Acta Psychiatr Scand. 1983;67:361-370.

23. Diener E, Emmons RA, Larsen RJ, Griffin S. The satisfaction with life scale. J Pers Assess 1985;49:71-75.

24. Fishbain DA, Pulikal A, Lewis JE, Gao J. Chronic pain types differ in their reported prevalence of post -traumatic stress disorder (PTSD) and there is consistent evidence that chronic pain is associated with PTSD: an evidence-Based structured systematic review. Pain Med. 2017;18:711-735.

25. Elliott DE, Bjelajac P, Fallot RD, Markoff LS, Reed BG. Traumainformed or trauma-denied: principles and implementation of traumainformed services for women. J Community Psychol. 2005;33:461-477.

26. Goldstein H, Safaeian P, Garrod K, Finamore P, Kellogg-Spadt S, Whitmore K. Depression, abuse and its relationship to interstitial cystitis. Int Urogynecol J. 2008;19:1683.

27. Dunleavy K, Kubo Slowik A. Emergence of delayed posttraumatic stress disorder symptoms related to sexual trauma: patient-centered and trauma-cognizant management by physical therapists. Phys Ther. 2012;92:339-351.

\section{SUPPORTING INFORMATION}

Additional Supporting Information may be found online in the supporting information tab for this article.
How to cite this article: McKernan LC, Johnson
BN, Reynolds WS, et al. Posttraumatic stress disorder in interstitial cystitis/bladder pain syndrome: Relationship to patient phenotype and clinical practice implications. Neurourology and Urodynamics. 2019;38:353-362. https://doi.org/10.1002/nau.23861 